\title{
Effect of ionizing radiation on acinar morphogenesis of human prostatic epithelial cells under three-dimensional culture conditions
}

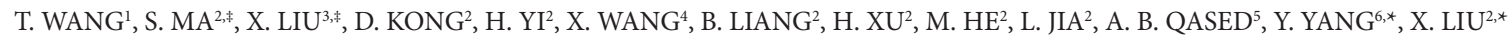 \\ ${ }^{1}$ Dept. Radiation Oncology, $2^{\text {nd }}$ Hospital affiliated to Jilin University, Changchun, 130021, China; ${ }^{2}$ Key Laboratory of Radiobiology (Ministry of \\ Health), School of Public Health, Jilin University, Changchun, 130021, China; ${ }^{3}$ Dept. Bioinformatics and Statistics, School of Public Health, Jilin \\ University, Changchun, 130021, China; ${ }^{4}$ Department of Radiology and Radiation Oncology, China-Japan Union Hospital, Changchun, 130021, \\ China; ${ }^{5}$ Dept. Surgery, $2^{\text {nd }}$ Hospital affiliated to Jilin University, Changchun, 130021, China; ${ }^{6}$ Dept. Oncology, $2^{\text {nd }}$ Hospital affiliated to Harbin \\ Medical University, Harbin, 150086, China
}

*Correspondence: liuxiaod@jlu.edu.cn,yangy@medmail.com.cn

${ }^{*}$ Contributed equally to this work.

Received October 6, 2011 / Accepted November 9, 2011

\begin{abstract}
Homeostasis is maintained by the interplay of multiple factors that directly or indirectly regulate cell proliferation and cell death. Complex multiple interactions between cells and the extracellular matrix occur during acinar morphogenesis and changes in these might indicate carcinogenesis of cells from a normal to a malignant, invasive phenotype. In this study, the human prostatic epithelial cell line RWPE-1 was cultured under three-dimensional (3-D) culture conditions, and the effect of ionizing radiation on acinar morphogenesis and its association with autophagy were discussed. The results illustrated that formation of specific spheroid (acinar) structures was detectable under 3-D culture conditions. Radiation induced the disruption of acini in different cell models using either gene overexpression (Akt) or gene knock-down (Beclin 1 and ATG7). Introduction of $A k t$ not only accelerated the growth of cells (i.e., caused the cells to manifest elongating and microspike-like structures that are obviously different from structures seen in wild-type RWPE-1 cells under two-dimensional conditions), but also changed their morphological characteristics under 3-D culture conditions. Knock-down of autophagy-related genes (Beclin 1 and ATG7) increased the radiosensitivity of cells under 3-D culture conditions, and cells died of non-apoptotic death after radiation. The results suggested that ionizing radiation may change the cell phenotype and the formation of acini. Additionally even the autophagy mechanism may play a role in these processes.
\end{abstract}

Key words: ionizing radiation, acinar, 3-D culture, autophagy

Prostate cancer is the most frequently diagnosed type of cancer in mature males [1]. Homeostasis in the prostate is maintained by the interplay of multiple factors that directly or indirectly regulate cell proliferation and cell death. Environmental stimuli could also result in transformation from normal prostatic cells to a malignant phenotype [2]. During the progression to a malignant, invasive state, cancer cells lose their normal cell organization, cell polarity, as well as cell-cell and cell-extracellular matrix (ECM) adhesion. The study of prostate cancer requires analysis of various factors that control normal acinar morphogenesis and their alteration in the carcinogenesis process. Further understanding of the mechanisms that control normal acinar morphogenesis or alterations that occur in the pathogenesis of prostate cancer is necessary. For such studies, appropriate in vitro cell model systems that mimic the process of acinar morphogenesis in a three-dimensional (3-D) microenvironment such as that found in vivo are needed.

Recently, 3-D culture has opened new directions for both research purposes and medical applications, including biochemical and architectural signaling in physiologically relevant contexts, recapitulation of developmental and pathological conditions in 3-D culture, and insights into mechanisms that control normal tissue formation $[3,4]$. The principle of 3-D culture is to place cells under conditions that induce formation of multicellular, tissue-like structures developing in the $\mathrm{X}, \mathrm{Y}$, and $\mathrm{Z}$ planes. These 3-D structures are characterized by establishment of adhesion complexes and tissue polarity, and by changes in cytoskeletal structure and cell volume that are significantly different from those found in cells cultured as 
monolayers (two-dimensional; 2-D). As a result, the phenotype, function, and regulation of signaling pathways under 2-D and 3-D culture conditions are fundamentally different. The science of 3-D cultures center around the recapitulation of physiological behaviors found in the organism, where reciprocal and dynamic cell-cell and cell-ECM interactions affect intercellular biochemical and structural signals, hence influencing gene expression and homeostasis. 3-D culture systems provide unique opportunities to mimic many aspects of developmental and pathological phenomena, to elucidate molecular mechanisms, and to produce tissues for transplantation purposes $[5,6]$.

In the present study, using non-tumorigenic human prostatic epithelial RWPE-1 cells, we describe a 3-D in vitro culture model to study the mechanisms involved in acinar morphogenesis in the absence or presence of genes associated with cell growth and death. RWPE-1 cells were subjected to both gene-overexpression $(A k t)$ and gene knock-down (Beclin 1 and ATG7) to establish cell models with different genetic backgrounds. Ionizing radiation (IR) was used as a model of environmental physical stress. We show in the present study that human prostatic epithelial cells were induced to display a nonapoptotic-like phenotype in an accumulative and dose-dependent manner. Using the 3-D culture system, we also provide direct experimental proof that IR changes gene expression profiles and consequently affect the cell phenotype, cell growth rate, and formation of acinar structures. Our results suggest that IR exposure can disrupt mammary morphogenesis and compromise growth regulation at the tissue architectural level.

\section{Materials and Methods}

Cell lines and materials. Human prostatic cells, RWPE-1, and modified RWPE cells that were established by retroviral infection of Beclin 1-shRNA, ATG7-shRNA, in our lab were used in the present study. The RWPE-1 cell line is derived from non-neoplastic human prostatic epithelial cells by immortalization with human papilloma virus 18 [7]. These cells mimic normal prostatic epithelial cell behavior in their response to growth factors and androgens and in their expression of prostate specific antigen and androgen receptor following androgen exposure; moreover, RWPE1 cells neither form tumors in nude mice nor do they are invasive [7].

Growth factor-reduced Matrigel and high concentration rat tail collagen I were purchased from BD Biosciences (San Diego, CA). Antibodies against different proteins were as follows: $a 6$ integrin was purchased from Chemicon (Temecula, $\mathrm{CA})$; anti-actin $\mathrm{mAb}$ and propidium iodide were purchased from Sigma (St Louis, MO); anti-Beclin $\mathrm{mAb}$ from BD Biosciences; anti-p53 (Ab6) from Oncogene (Cambridge,MA); anti-p21waf1(Ab1) from Calbiochem; anti-phospho-Akt (Ser473) mAb from Cell Signaling (Beverly, MA); Alexa Fluor488-labeled goat anti-rat and anti-mouse immunoglobulin
G (IgG) from Molecular Probes (Eugene, OR); pSUPER retroviral vector from OligoEngine (Seattle, WA).

2-D cell culture. RWPE cells were maintained in basal keratinocyte serum-free medium (KSFM; Life Technologies, Paisley, UK) supplemented with $50 \mathrm{mg} / \mathrm{ml}$ bovine pituitary extract (BPE), $5 \mu \mathrm{g} / \mathrm{ml}$ epidermal growth factor (EGF), and $1 \%$ antibiotic-antimycotic mixture, referred to as complete KSFM. For passaging, cells were washed with $\mathrm{Ca}^{2+} / \mathrm{Mg}^{2+}$-free phosphate-buffered saline (PBS), and then incubated with a trypsin-EDTA mixture (0.05\% trypsin, $0.53 \mathrm{mM}$ EDTA) for $5-15 \mathrm{~min}$ in a $37^{\circ} \mathrm{C}$ incubator. Cells were dislodged by tapping, neutralized with $2 \%$ calf serum in PBS, recovered by centrifugation, and counted and seeded in plates or eight-well chamber slides.

3-D cell culture. The 3-D culture method was modified from an overlay 3-D culture method described previously [8]. RWPE cells were maintained in complete KSFM media containing $2 \%$ calf serum for confluence, after which they were trypsinized and harvested in serum-free trypsin-EDTA mixture, and then re-suspended in assay media (DMEM/ F12 supplemented media plus $2 \%$ horse serum, $0.5 \mu \mathrm{g} / \mathrm{ml}$ hydrocortisone, $100 \mathrm{ng} / \mathrm{ml}$ cholera toxin, $10 \mu \mathrm{g} / \mathrm{ml}$ insulin, and $1 \times$ antibiotic-antimycotic mixture). Cells $\left(2000 / \mathrm{cm}^{2}\right)$ were then diluted in assay media plus $2 \%$ growth factorreduced Matrigel and seeded in eight-well chamber slides (Nalge Nunc, Naperville, IL), which were pre-coated with $40 \mu \mathrm{l}$ of growth factor-reduced Matrigel and type I collagen mixture (3:1 ratio) for morphological and immunofluorescence analysis. Type I collagen (final concentration, $2.0 \mathrm{mg} / \mathrm{ml}$ ) was included in the matrix to facilitate acquisition of spindle-shaped morphology and migration of cells in three dimensions, and to simultaneously dilute the EGF concentration in the Matrigel. The cultures were incubated in $5 \% \mathrm{CO}_{2}$ at $37^{\circ} \mathrm{C}$ and were replenished with fresh medium every 3 days for a week followed by more frequent medium changes (every 2 days) thereafter.

Ionizing radiation treatment. Ionizing radiation was administered with a cobalt 60 irradiator. The dose rate used was $1.25 \mathrm{~Gy} / \mathrm{min}$. Cells were treated at room temperature with total absorption doses of $2 \mathrm{~Gy}$ or $5 \mathrm{~Gy}, 24 \mathrm{~h}$ after the initial plating onto culture plates or chamber slides. Cells were harvested at different time intervals depending on different experimental design strategies.

Retrovirus-mediated gene transfer and gene silencing. Amphotrophic retrovirus was produced by transfecting pLZRS.neo Akt - Mu(K179M) and pLZRS.neo Akt cDNAinto 293 T cells. Ampho Pack vector was co-transfected using a standard calcium phosphate co-precipitation procedure. RWPE cells were then infected with a retroviral soup containing viral particles and the populations were selected using $1 \mathrm{mg} / \mathrm{mL}$ G418 (Invitrogen, Carlsbad, CA). Stable down-regulation of Beclin-1 and ATG-7 was achieved using a retrovirus-mediated RNA interference system established in our laboratory at Harvard School of Public Health, using oligonucleotide sequences previously described [9]. The 
procedure is as follows: Briefly, 293T packaging cells were transfected with $10 \mu \mathrm{g}$ of pSUPER vector, together with 10 $\mu \mathrm{g}$ of Ampho Pack, the packaging vector, using a standard calcium phosphate co-precipitation procedure. The precipitate was removed and fresh media were added to the transfected $293 \mathrm{~T}$ cells $12 \mathrm{~h}$ after transfection. After $24 \mathrm{~h}$, the transfected $293 \mathrm{~T}$ cells were placed into a $32^{\circ} \mathrm{C}, 5 \% \mathrm{CO}_{2}$ incubator for another $48 \mathrm{~h}$, and then culture supernatants were collected and filtered. Prostatic cells were infected with the filtered viral supernatants in the presence of $8 \mu \mathrm{g} / \mathrm{ml}$ polybrene (Sigma) for $3 \mathrm{~h}$, after which the medium was changed for fresh medium. Infected cells were selected with antibiotics after confluence. Infected cell populations were selected using $1 \mu \mathrm{g} / \mathrm{ml}$ puromycin.

Western blot analysis. Total protein extracts from RWPE cells were fractionated by gel electrophoresis, and proteins were transferred to nitrocellulose membranes. The following primary antibodies were used: anti-Beclin 1, anti-p53, antip21waf1, and anti-actin. The protein-antibody complexes were detected using horseradish peroxidase-conjugated secondary antibodies followed by enhanced chemiluminescence western blotting detection reagents (Amersham, Arlington Heights, IL).

Immunofluorescence analysis and confocal microscopy detection. For immunocytochemistry, slides were fixed with formalin for $30 \mathrm{~min}$ at room temperature followed by permeabilization with $0.5 \%$ Triton X-100, and then two blocking steps were used for 3-D immunostaining. After blocking in IF buffer $\left(130 \mathrm{mM} \mathrm{NaCl}, 7 \mathrm{mM} \mathrm{Na}_{2} \mathrm{HPO}_{4}, 3.5 \mathrm{mM} \mathrm{NaH} \mathrm{PO}_{4}, 7.7 \mathrm{mM}\right.$ $\mathrm{NaN}_{3}, 0.1 \%$ bovine serum albumin (BSA), $0.2 \%$ Triton X-100, and $0.05 \%$ Tween-20) supplemented with $10 \%$ normal goat serum for $1 \mathrm{~h}$ at room temperature, slides were secondarily blocked with the blocking buffer mentioned above, except that $20 \mu \mathrm{g} / \mathrm{ml}$ goat anti-mouse $\mathrm{F}\left(\mathrm{ab}^{\prime}\right) 2$ fragment (\#115-006-006, Jackson ImmunoResearch, West Grove, PA, USA )was added. Samples were then incubated with primary antibodies. As the secondary antibody, Alexa Fluor 488-labeled goat anti-mouse was used together with 4,6-diamidino-2-phenylindole (DAPI). Slides were mounted with fluorescent mounting medium (Dako, Houston, TX) and examined using a fluorescence microscope.

For confocal analysis, the 3-D structures were fixed, immunostained as mentioned above, and examined using a Leica TCSNT confocal laser scanning microscope (Chidren's Hospital, Boston, MA). For the selected 3-D structures, serial optical sections were collected from the apical surface at $0.5-\mu \mathrm{m}$ step intervals and rendered in three dimensions using VoxelView software (Vital Images, Minnetonka, MN) to examine the spatial morphology of human prostatic epithelial cells.

Colony formation assay. The response of cells to irradiation was determined using the colony formation assay. Cells at $80 \%$ confluence were trypsinized and single-cell suspensions were obtained. The cells were plated in six-well plates at a density of 400 cells per well, and then exposed to different dosages of irradiation. Ten days later, cells were fixed with $70 \%$ ethanol for $15 \mathrm{~min}$ and stained with 10\% (v/v) Giemsa (Merck, Damstadt, Germany). Colonies consisting of more than 50 cells were counted as surviving colonies. Each experiment was performed in triplicate and the mean values \pm standard error of the mean (SEM) were presented.

Flow cytometry for cell cycle analysis. For cell cycle studies, cells were seeded at a density of $1 \times 10^{7} /$ dish and treated with total absorption doses of $2 \mathrm{~Gy}$ or $5 \mathrm{~Gy}$ of irradiation. Eighteen or twenty-four hours later, cell were fixed in cold $70 \%$ ethyl alcohol for at least $30 \mathrm{~min}$ and washed. The supernatant was then discarded and the pellet was re-suspended in $0.5 \mathrm{ml}$ of $50 \mathrm{mM}$ sodium citrate containing $0.1 \mathrm{mg} / \mathrm{ml} \mathrm{RNase} A$ at $37^{\circ} \mathrm{C}$ for $2 \mathrm{~h}$. Cell were stained with propidium iodide $(4 \mu \mathrm{g} / \mathrm{ml})$ at $4^{\circ} \mathrm{C}$. Distribution of cell cycle phases with different DNA content were analyzed by flow cytometry using a fluorescence -activated cell sorter (FACS) machine (Becton Dickinson, Franklin Lakes, NJ).

MTT assay. Growth analysis of cells was performed using the 3-(4,5-dimethylthiazol -2-yl)-2,5- diphenyl-terazolium bromide (MTT) assay. Cells were seeded in 96-well plates at a density of $4 \times 10^{5}$ cells/well. At different time points during incubation, MTT was added to each cell, the plates were further incubated at $37^{\circ} \mathrm{C}$ for $4 \mathrm{~h}$, and the formazan complex in each well was dissolved in $200 \mu$ dimethyl sulfoxide. Viable cells were detected by measuring the absorbance at $540 \mathrm{~nm}$.

Statistical analysis. Statistical analysis was performed using one-way analysis of variance followed by the unpaired $t$-test. Each assay was performed in triplicate and all data were presented as the mean \pm standard deviation (SD). A $P$-value $<0.05$ was considered to be statistically significant.

\section{Results}

Acinar morphogenesis under 3-D culture conditions. Three-dimensional cell cultures mimic physiologically relevant processes and thus offer various possible investigation strategies regarding molecular mechanisms of regulation. Under 3-D culture conditions, epithelial and endothelial cells can be induced to form multicellular, tissue-like acinar structures, which are characterized by establishment of spheroid adhesion clusters and tissue polarity with the central lumen significantly different from those found in cells cultured as monolayers (2-D) [10]. RWPE-1 cells are non-neoplastic human prostatic epithelial cells that can neither form tumors in nude mice nor are invasive [2]. In the present study, cells were stained for expression of a 6 integrin, which correlates with the ability to polarize and form acini. Nuclei were also stained with DAPI (blue fluorescence), and integrin distribution (green fluorescence) was indicative of typical acinar structures, including cell polarity and the central lumen as indicated in Figure 1 by both fluorescence microscopy and confocal microscopy.

Effects of radiation on acinar morphogenesis in cell models with Beclin 1 or ATG 7 gene silencing. Beclin 1 and ATG7 genes have been reported to play important roles in the process of autophagy $[9,11,12]$. In the current study, we man- 

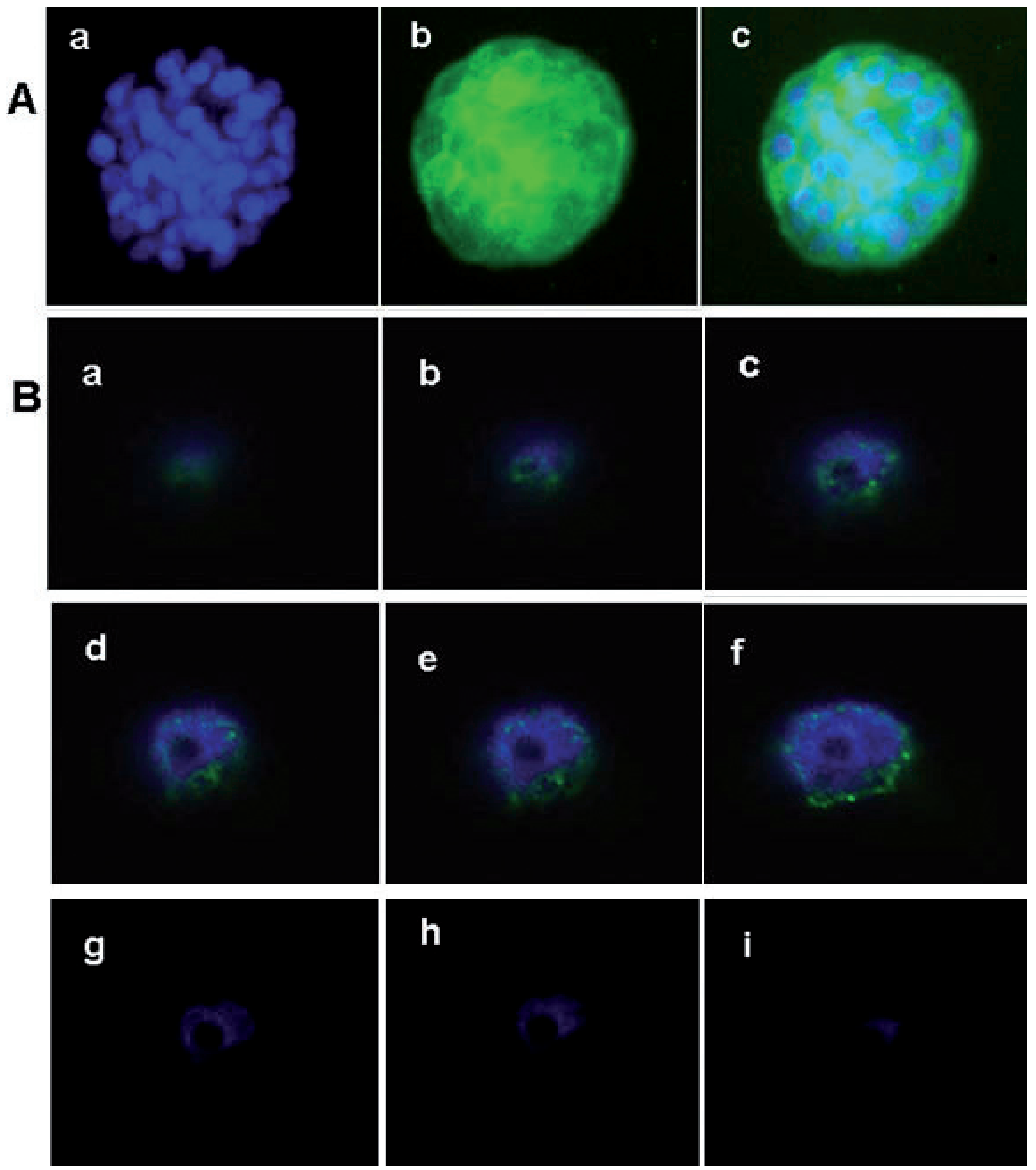

Figure 1. The spheroid structure of human prostatic epithelial cells under 3-D culture conditions. RWPE-1 cells were fixed and stained on the $10^{\text {th }}$ day after plating under 3-D culture conditions. A) Immunostaining results were analyzed by fluorescence microscopy. Nuclei (a, blue) and integrin (b, green) were stained, cells aggregated to form a spheroid structure, and the polarity of cells was visible (c, merged picture). B) Results from confocal analysis. Pictures of samples were taken every $0.5 \mu \mathrm{m}$ from top to bottom (a to $\mathrm{i}$ ). The outer layer (green) of integrin represents the polarity of cells, the blue-stained nuclei were wrapped inside, and the central lumen was visible. 


\section{Control}

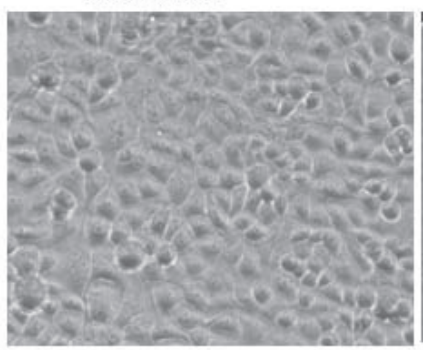

Beclin-RNAi

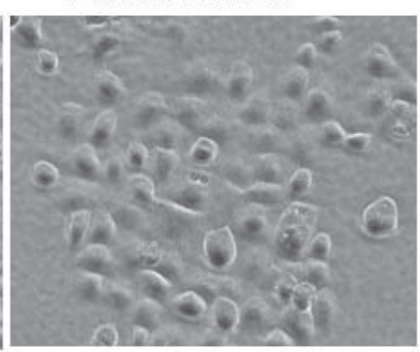

ATG7-RNAi

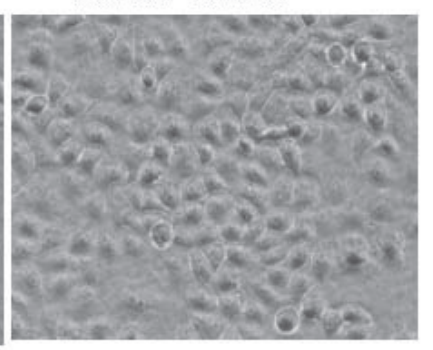

A

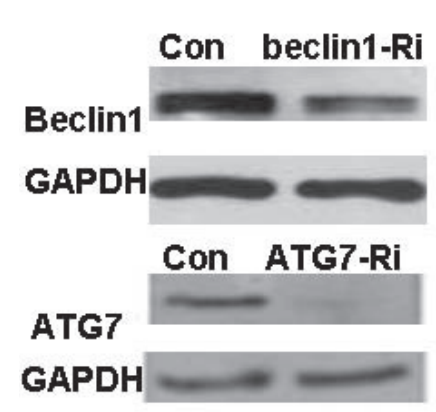

B
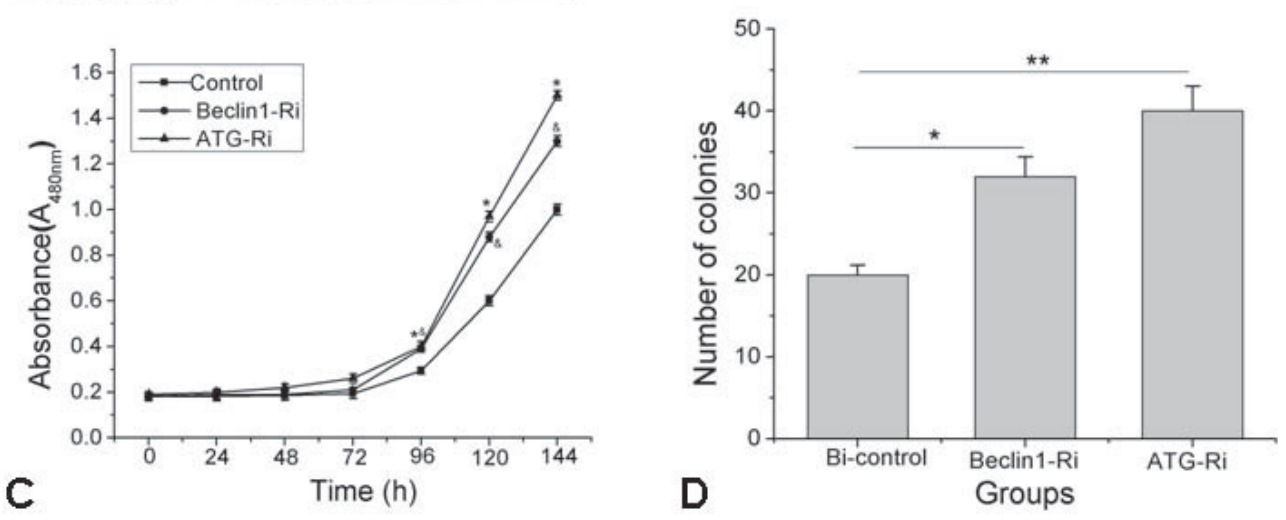

Figure 2. Changes of Mophological character and radiosensitivity in Beclin-/- and ATG7-/- cell model.

Beclin-ShRNA and ATG7-shRNA were introduced into RWPE-1 cells and the stable colonies were selected by using $1 \mu \mathrm{g} / \mathrm{mL}$ puromycin. A: phase-contrast detection of morphological changes in Beclin-/- and ATG7-/- cell model. B: western blot was used to verify the establishment of Beclin-/- and ATG7/- cell models under 2-dimentional culture. C: MTT assay was used to detect the growth of cells and the growth curve was made. D: colony formation assay was used to detect the long-term growth status.

aged to determine the effects of radiation on acini formation and possible functions of Beclin 1 and ATG7. The results are shown both under 2-D and 3-D culture conditions.

Establishment of cell models with Beclin-RNAi or ATG 7-RNAi and general parameter detection under 2-D and 3-D culture conditions. Beclin-shRNA and ATG7-shRNA were introduced into RWPE-1 cells and stable colonies were selected using $1 \mu \mathrm{g} / \mathrm{ml}$ puromycin. The cell models were then verified by western blot detection (Fig. 2). Morphological changes under 2-D culture conditions showed that Beclin-RNAi cells became round and the extension of twigs disappeared compared with RWPE-1 wild-type cells. However, ATG7RNAi cells retained the normal elongated morphological structure. Both Beclin-RNAi and ATG7-RNAi caused cells to grow faster, implying growth suppression of autophagy in RWPE-1 cells (Fig. 2).

5 -fluorouracil (FU) was used as a chemical control to compare with the physical stimulus of radiation, and protein expression was measured in both cases. Radiation was clearly seen to increase the expression of Beclin 1 and p21 in wild-type cells; 5-FU functioned in almost the same manner. After introduction of Beclin-shRNA or ATG7-shRNA using retroviral vectors, suppression of Beclin 1 and an increase in p21 was seen, whereas p53 showed no change. After radiation, Beclin 1 increased more significantly in Beclin-RNAi cells than in wild-type cells, while p21 showed no difference compared with wild-type cells (Fig. 3).

Effects of radiation on autophagy and radiosensitization in Beclin ${ }^{-1}$ and ATG7/- cells. Microtubule-associate protein light chain 3 (MAP-LC3) is a specific marker for autophagy and the Beclin gene can also be used to indirectly detect autophagic status. In this study, GFP-LC3 was introduced into cells transiently to observe its re-localization. The results showed that the appearance and aggregation of green punctate foci were visible in the cytosol $16 \mathrm{~h}$ after radiation (Fig. 4A). Beclin and MAPLC3 expression showed a time-dependent increase, particularly $16 \mathrm{~h}$ after radiation (Fig. 4B). The colony formation assay (Fig. 4C) showed that Beclin-RNAi and ATG7-RNAi led to increase of cell growth and proliferation (vs. control), radiation decreased the colony numbers in the three groups, but the extent in Beclin ${ }^{-1-}$ and ATG7 $7^{-1-}$ cells were much more striking. The surviving fraction curve (Fig. 4D) showed that the slope in Beclin ${ }^{-/}$and ATG7 ${ }^{-1-}$ cells were greater than the wild-type control (no difference between Beclin ${ }^{-1}$ and ATG7- cells), suggesting radiosensitization of Beclin-RNAi and ATG7-RNAi.

Changes in the cell cycle in Beclin-RNAi cell models. Flow cytometry was used to detect changes in the cell cycle after radiation. Two doses ( 2 and $5 \mathrm{~Gy}$ ) and two time points (18 and $24 \mathrm{~h}$ ) were selected in the present study. The histogram 


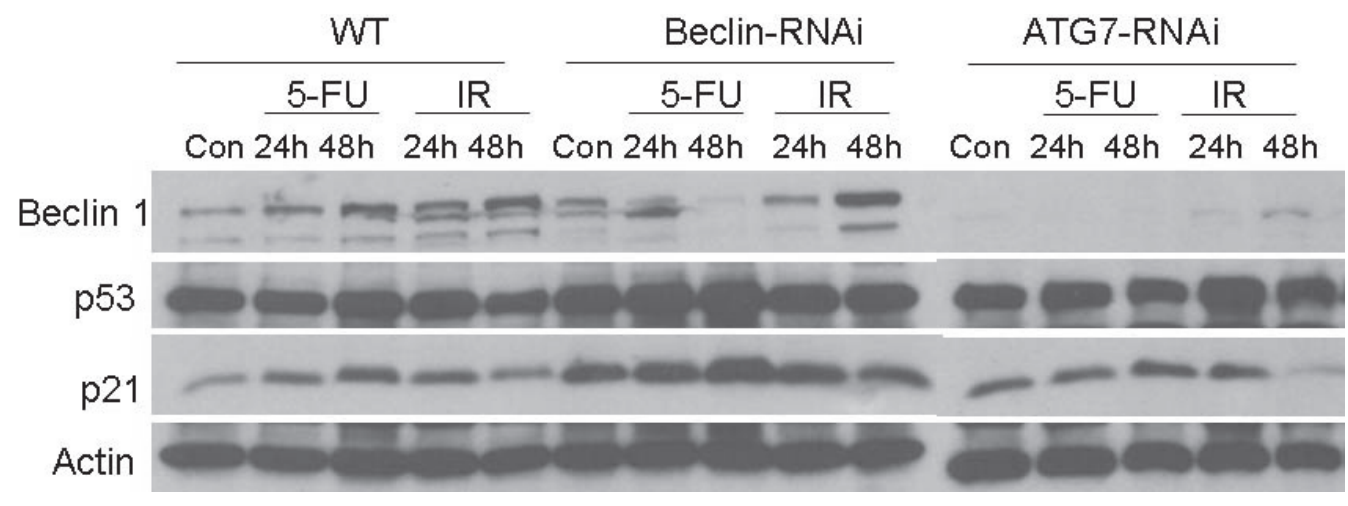

Figure 3. The protein expression profile in gene knock-down cells by western blot.

The RWPE-1 cells were infected by Beclin-RNAi and ATG7-RNAi retroviral vectors and the cell models with different genetic background were established. The cells were harvested and proteins were extracted at either $24 \mathrm{~h}$ or $48 \mathrm{~h}$ after the treatments. Beclin 1, p53, p21 were detected by western blot, and actin was used as the internal control. 5-FU was also used as a chemical control in this study.

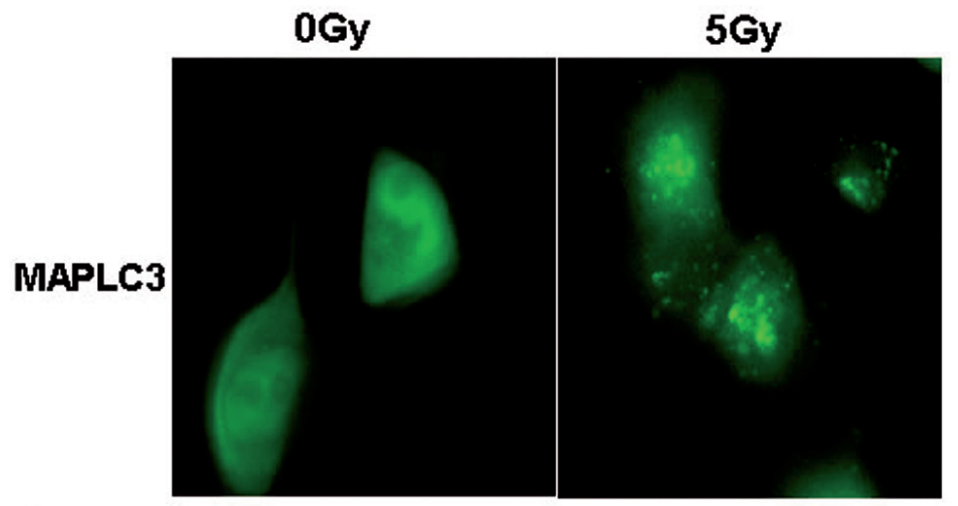

A
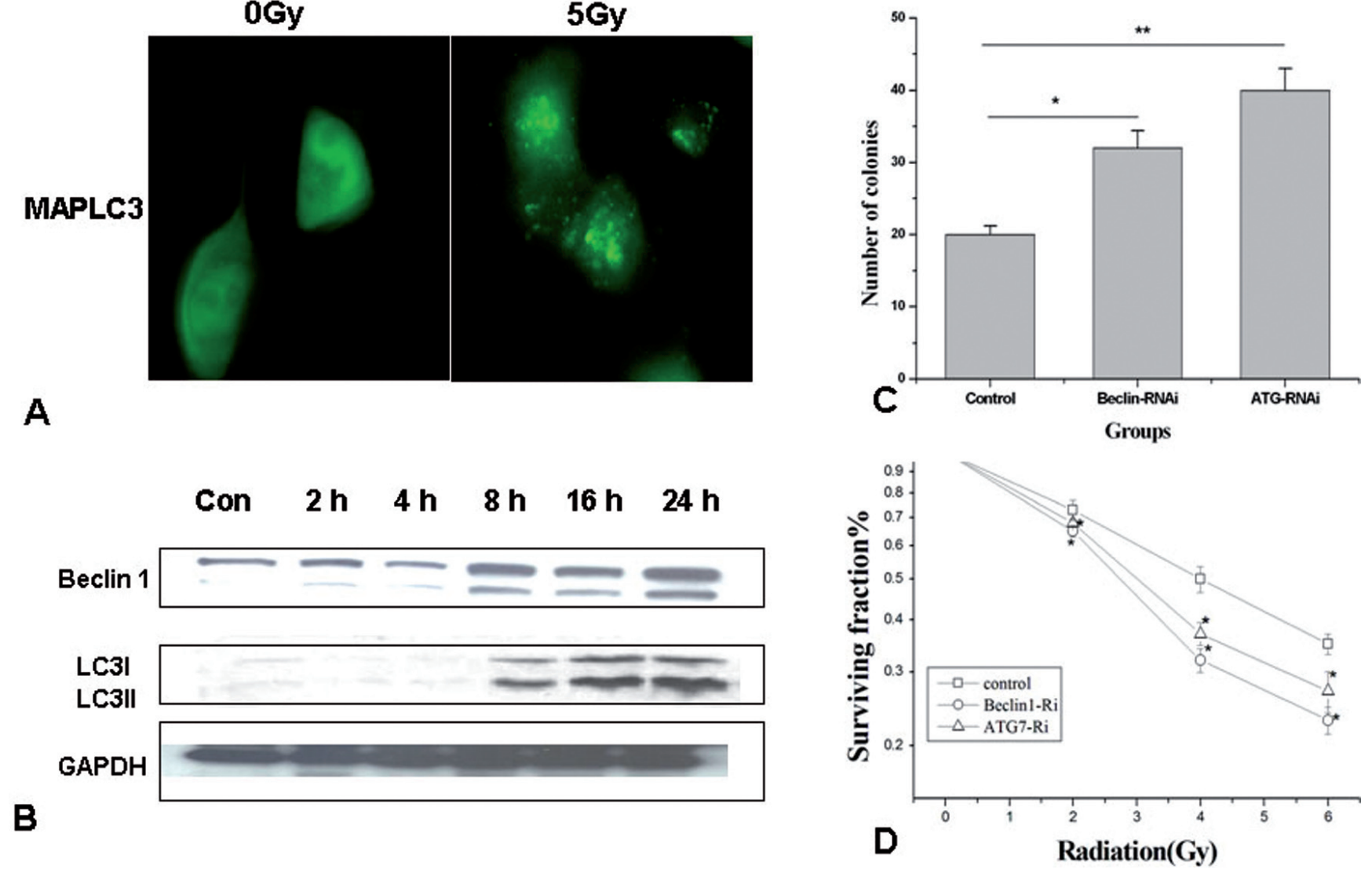

Figure 4. The roles of Beclin in radiation-induced autophagy. Microtubule-associate protein light chain 3 (MAP-LC3) is a specific marker for autophagy and the Beclin gene is also used to indirectly detect autophagic status. A) GFP-LC3 was introduced into cells transiently to observe its re-localization. The appearance and aggregation of green punctate foci in the cytosol suggest the autophagy process after radiation. B) Time-course changes of Beclin and MAP-LC3 expression were detected by western blot after radiation. C) The colony formation assay was used to detect changes in cell growth and proliferation. D) The MTT assay was used to detect cell growth and the surviving fraction curve was constructed.

in Fig. 5 showed that the control group manifested G1 arrest, G2 arrest, and $\mathrm{S}$ phase decrease after radiation, confirming the radiobiological rules that radiation induces $\mathrm{G} 1$ and $\mathrm{G} 2$ arrest and provides a chance to repair damaged DNA. However, in the Beclin-RNAi group, radiation triggered G1 decrease, G2 arrest, and S phase extension, suggesting that the lack of au- 

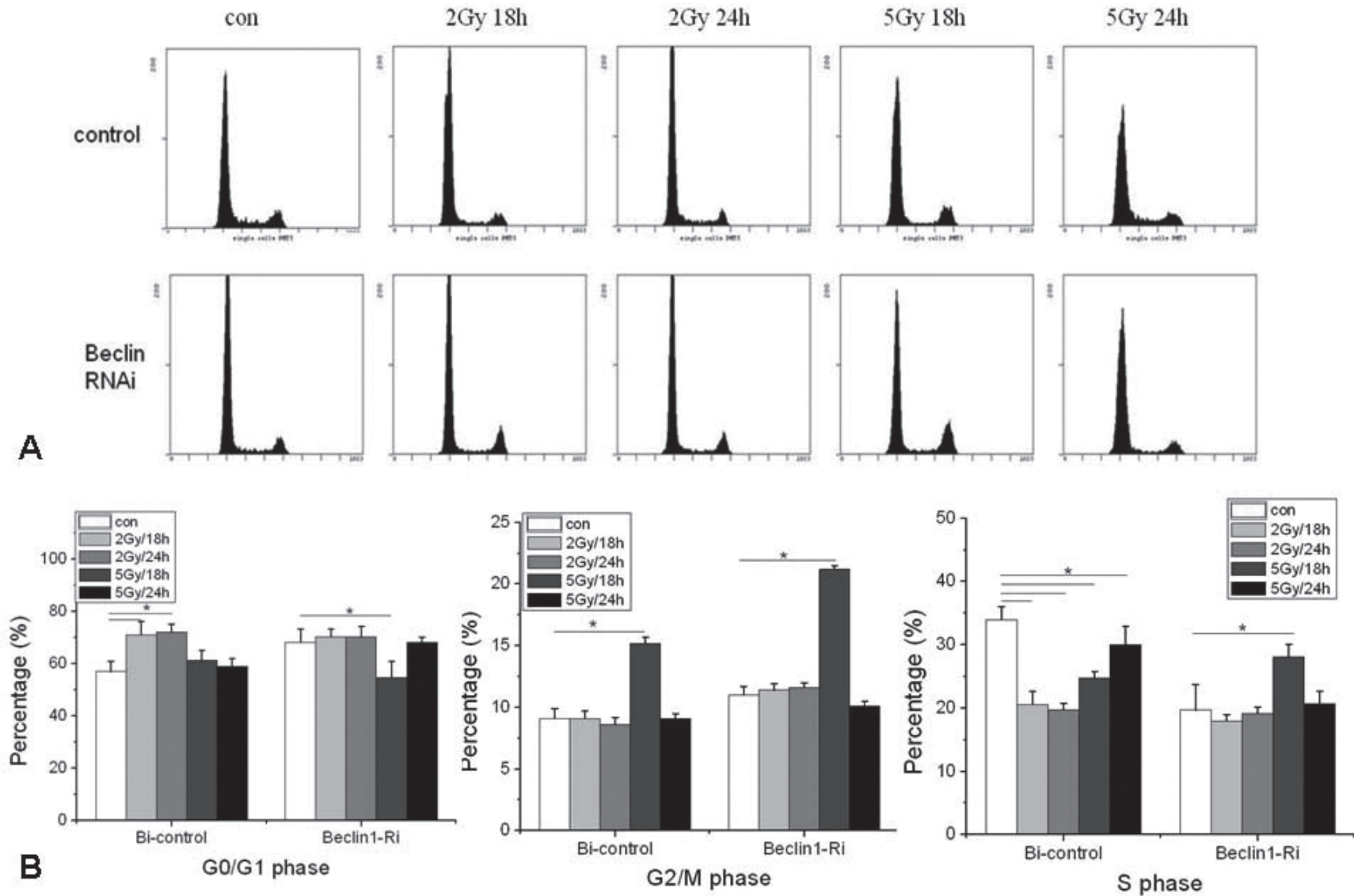

Figure 5. Changes in the cell cycle progression in Beclin ${ }^{-/}$cells after radiation.

Flow cytometry was used to detect changes in the cell cycle after radiation. Two doses ( 2 and $5 \mathrm{~Gy}$ ) and two time points ( 18 and $24 \mathrm{~h}$ ) were selected. A) Histogram of Beclin ${ }^{-/}$cells after treatment with radiation. B) Statistical analysis of the flow cytometry histogram, showed G1 arrest, G2 arrest, and S phase decrease in the control group (wild type) after radiation; however, in the Beclin ${ }^{-/}$group, radiation triggered G1 decrease, G2 arrest, and S phase extension.

tophagy resulting from Beclin-RNAi might cause deletion of the G1 checkpoint and result in cells going into $S$ phase. Thus, the replication of abnormal DNA in S phase and accomplishment of cycle progression through G2/M phase might lead to genome instability.

Changes in acinar morphogenesis in Beclin-RNAi and ATG7RNAi cell models under 3-D culture conditions.

The morphological changes in gene knock-down RWPE cells by radiation under 3-D culture conditions were detected by immunostaining methods described previously. Fig. 6 showed that wild-type RWPE cells aggregated and formed acini under 3-D culture conditions, with clearly defined cell polarity, whereas in Beclin-RNAi and ATG7-RNAi cells, although the cells were clustered together, normal acini were not observed. After treatment with radiation, either 2 or $5 \mathrm{~Gy}$, cells detached from each other, and cell death was more obvious in Beclin-RNAi and ATG7-RNAi cells than in wild-type cells, suggesting that disruption of the autophagy process blocked acinar morphogenesis under 3-D culture conditions (Fig. 6).
Effects of radiation on acinar morphogenesis in cells overexpressing Akt. Akt has been shown to play critical roles in regulation of multiple cellular functions such as transcription, cell survival, cell cycle progression, angiogenesis, and cell motility; additionally, the Akt gene was recently reported to be associated with a novel type of cell death-autophagy [13]. In the current study, wild-type Akt (Wt-Akt) and mutant Akt (179M, Mu-Akt) were introduced into RWPE cells, respectively, and the morphological characteristics, protein expression, and cell cycle progression under both 2-D and 3-D culture conditions were observed.

Establishment of cell models with Akt overexpression and general parameter detection under 2-D culture conditions. The growth status of RWPE with or without transfection using either Wt-Akt or Mu-Akt under 2-D culture conditions was detected. RWPE cells with introduction of Wt-Akt elongated greatly and formed microspike-like structures; however, $\mathrm{Mu}$-Akt altered the morphology and formed microspikes, but to a lesser extent. The growth rate was detected using both the growth curve of RWPE cells with different gene 

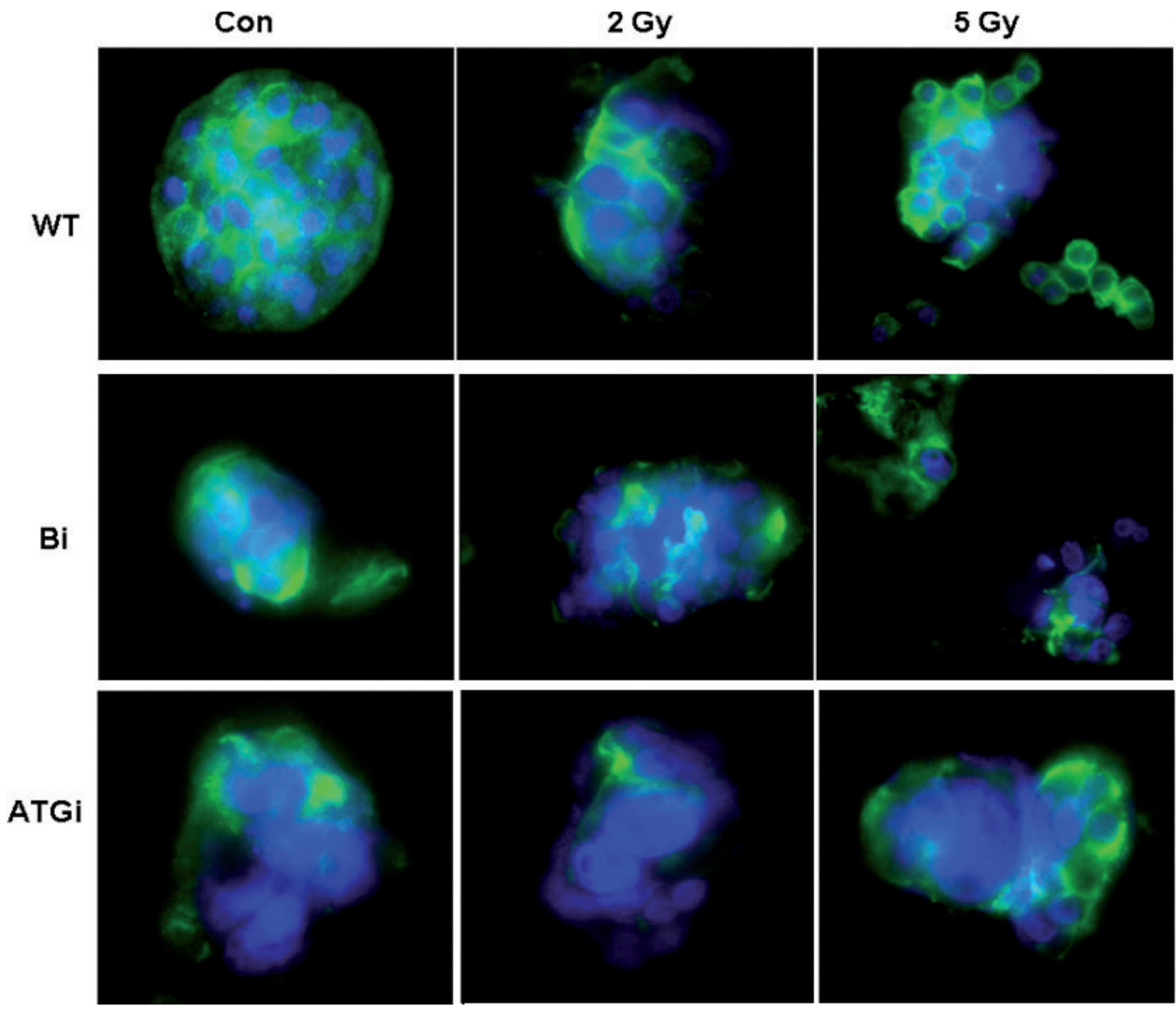

Figure 6. Morphological changes in gene knock-down RWPE cells by radiation under 3-D culture conditions. Gene knock-down cell models were fixed and stained on the $\mathbf{1 0}^{\text {th }}$ day after plating under 3-D culture conditions. Nuclei (blue) and integrin (green) were stained and the samples were analyzed by fluorescence microscopy. The wild-type RWPE cells aggregated and formed acini under 3-D culture conditions, whereas cells treated with BeclinRNAi and ATG7-RNAi were able to cluster together but did not form normal acini: cells were merely organized into a large cell mass and cell death was visible. After treatment with either 2 or 5 Gy radiation, the structure was disrupted, cells detached from each other, and cell death was more obvious in Beclin-RNAi and ATG7-RNAi cells than in RWPE wild-type cells.

expression profiles and the colony formation assay (Fig. 7). Cells transfected with the wild-type or mutant $A k t$ gene grew faster than wild-type cells and empty vector-infected cells (even the empty retroviral vector stimulated cell growth), illustrating that Akt functions as a growth-related gene in RWPE cells.

The protein expression profile was analyzed and anti-Akt and phospho-Akt (Ser 273) were used to blot the proteins. Introduction of either Wt-Akt or Mu-Akt dramatically increased expression of Akt. Phospho-Akt was detected only in cells containing Wt-Akt introduction, and its expression was unaltered with or without radiation treatment (Fig. 8).

Cell cycle changes caused by radiation in Akt-overexpressing cell models. The role of Akt as a growth-related gene was confirmed because, in the absence of radiation, the $179 \mathrm{M}$ and Akt groups showed higher growth levels than the control group (Fig. 9A). After radiation, the number of colonies decreased, compared with the mock-irradiation group. Cell cycle progression was detected by flow cytometry (Fig. 9B and 

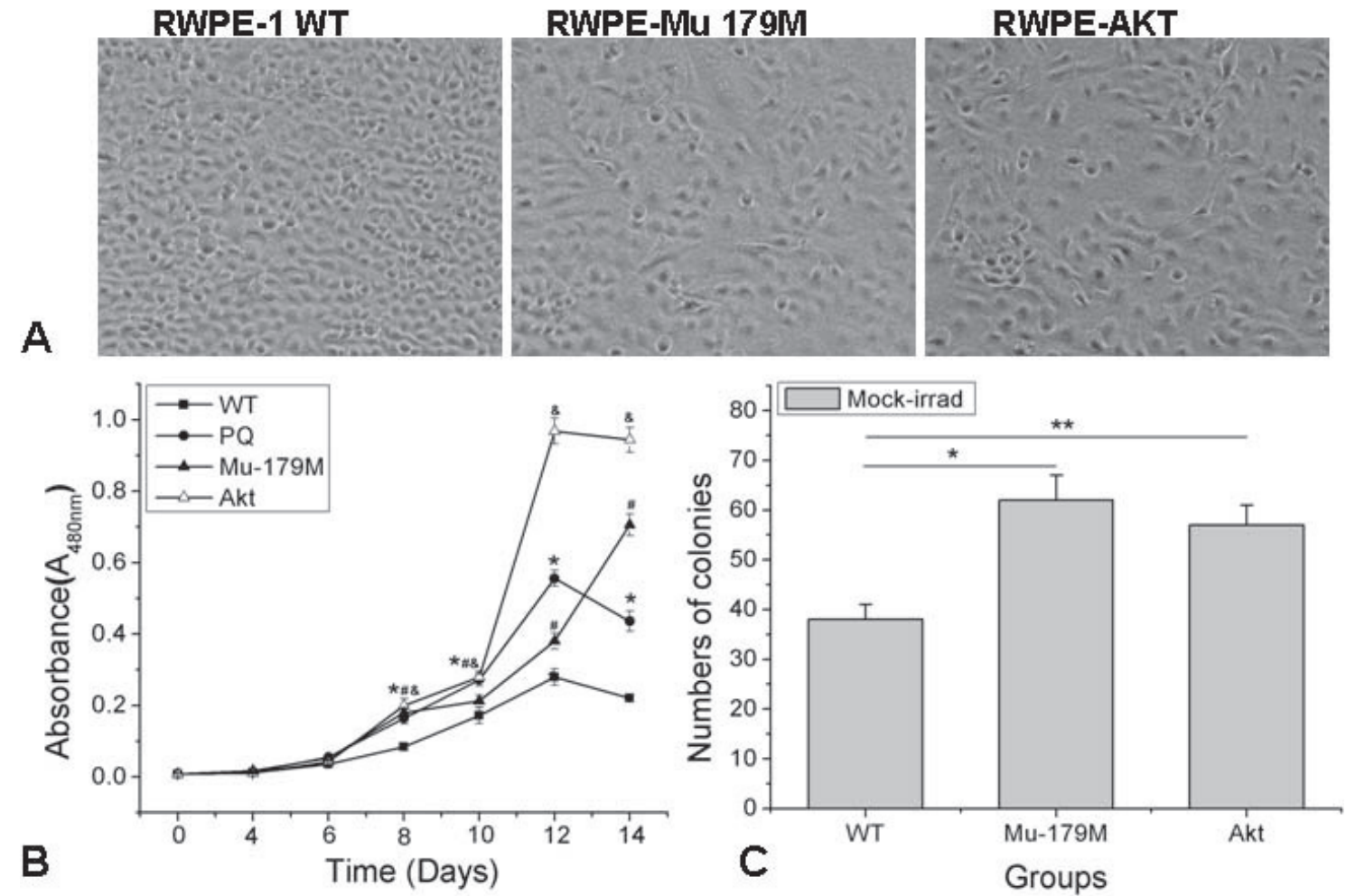

Figure 7. The Changes of Morphological character and growth status in cell model with Akt overexpression.

Akt and Akt mutant (179M) were transfected into cells to establish the models with different Akt expression background, while the same empty vector was transfected in wt RWPE-1 cells as a control. The proteins were extracted at either 24 hrs or 48 hrs after radiation, anti-Akt and phospho-Akt(Ser 273 ) were used to blot the proteins and actin was used as the internal control. A: phase-contrast detection of morphological changes in Akt overexpression cell model. B: MTT assay was used to detect the growth of cells and the growth curve was made. C: colony formation assay was used to detect the long-term growth status.

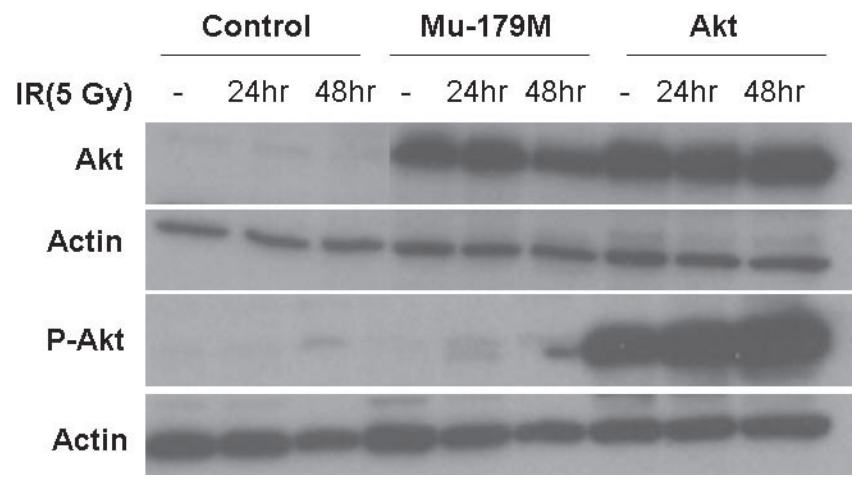

Figure 8. The protein expression profile in Akt-overexpression cells by western blot.

The protein expression profile was analysized in cell models with different Akt expression background (wild-type and 179M) by western blot. Proteins were extracted at either $24 \mathrm{hr}$ or $48 \mathrm{hr}$ after radiation, anti-Akt and phospho-Akt(Ser 273 ) were used to blot the proteins, and actin was used as the internal control. The constitutive expression of Akt was very weak in control, while the introduction of either Wt-Akt or Mu-Akt increased the expression of Akt dramatically. The phosph-Akt could only be detected in the cells with Wt-Akt introduction, the expression of phosphor-Akt shown no differences with or without radiation treatment.
C), and showed that lower dose of radiation (2 Gy) caused G1 arrest, while a higher dose ( $5 \mathrm{~Gy}$ ) caused G2 arrest. No difference was found between the control and Akt overexpression groups; therefore no function of Akt was demonstrated in the cell cycle here.

Changes in acinar morphogenesis in Akt-overexpressing cells under 3-D culture conditions. The morphological characteristics of cells under 3-D culture conditions were observed by immunostaining (Fig. 10). Nuclei (blue) and integrin (green) were stained and samples were analyzed by fluorescence microscopy. Wild-type RWPE cells aggregated and the formation of acini was visible under 3-D culture conditions with clearly defined cell polarity. However, in the Wt-Akt or Mu-Akt group, although cells were clustered together, normal acini were not observed. After $5 \mathrm{~Gy}$ of radiation, cells exhibited a disorganized structure and turned brighter and smoother; no cell death was visible in both Wt-Akt- and Mu-Akt-infected cells.

\section{Discussion}

Radiation-induced cell death, predominantly reported to occur in an apoptotic manner $[14,15]$, has been implicated 


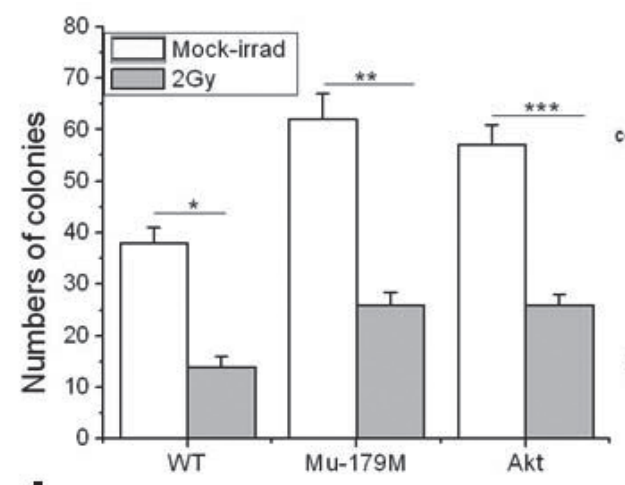

A

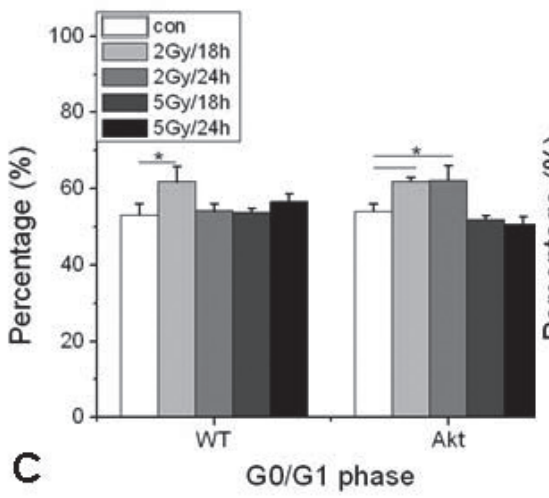

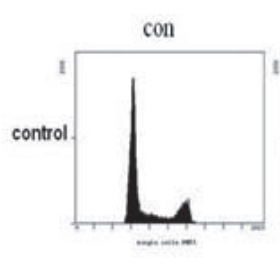
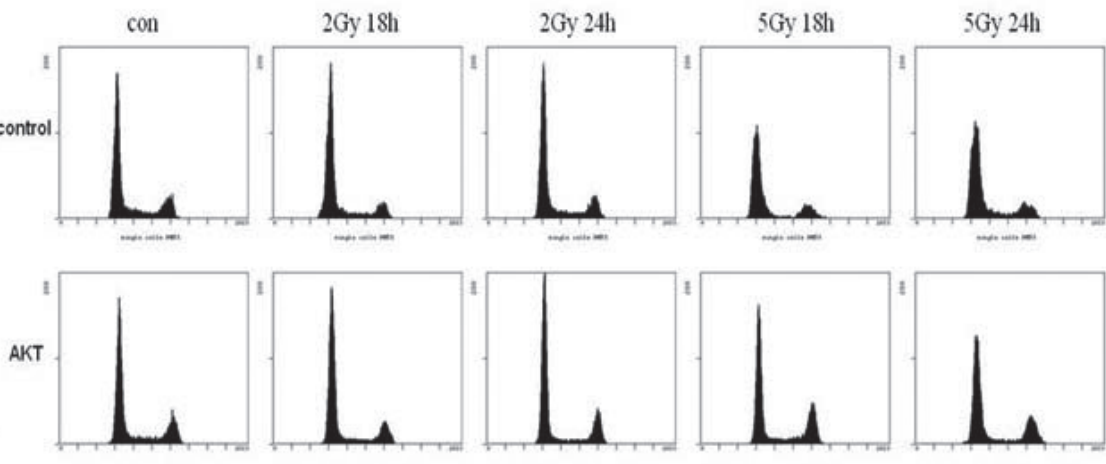

B
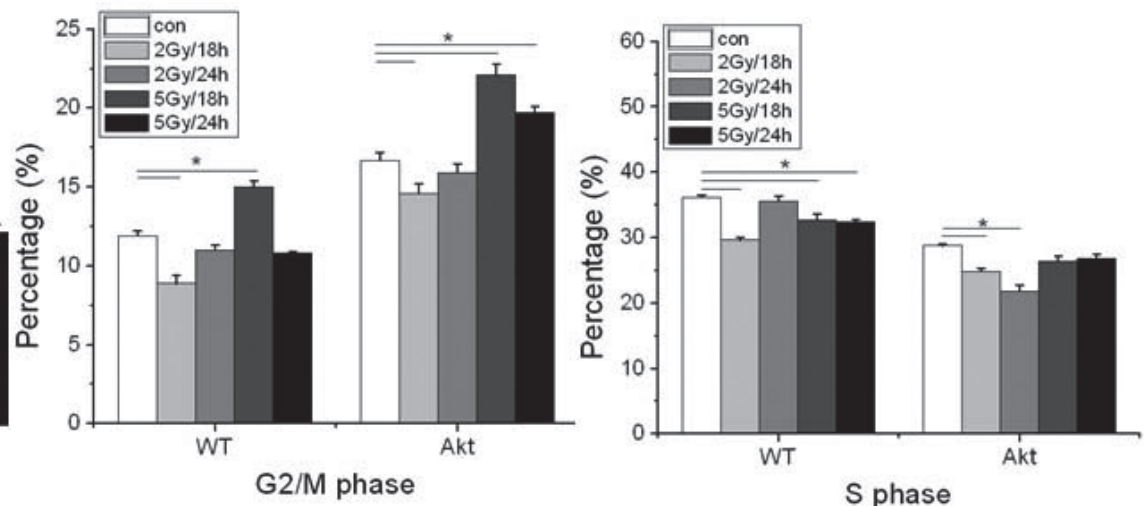

Figure 9. Changes in cell growth and cell cycle progression in Akt-overexpressing cells after radiation. A) The colony formation assay was used to detect the long-term growth status of Akt-overexpressing cells after radiation. B) Histogram of Akt-overexpressing cells after treatment with radiation by flow cytometry. C) Statistical analysis of the flow cytometric histogram showed that a lower dose of radiation (2 Gy) caused G1 arrest, while a higher dose (5 Gy) caused G2 arrest. Additionally, no difference was found between the control and Akt overexpression groups.

in cancer treatment. Here, we provide a novel mechanism by which IRcan deregulate cell-cell and cell-ECM interactions and perturb acinar morphogenesis under 3-D culture conditions, consequently fostering formation of an oncogenic environment. A non-apoptotic type of cell death, autophagy, might play an important role in this process.

Traditional tissue culture, referred to as monolayer or 2D cultures, has permitted numerous discoveries, including identification of different components of the cell machinery and regulatory principles of fundamental cellular events such as proliferation, adhesion, and cell death [6]. However, almost all cells change their function dramatically in such cultures compared with in vivo situations. Moreover, differentiated normal cells essentially lose their ability to express tissue-specific genes. That the signaling pathways are regulated differently in 3-D compared with 2 -D cultures $[5,16,17]$, is now highly appreciated.

The 3-D culture system mimics the microenvironment in vivo and in the ECM, provided that, according to different tissue types, it is able to ensure both cell shape and chemistry to the maximum extent. Here, the 3-D model allowed us to recapitulate at the cellular level the process of acinar morphogenesis in vitro.

In this study, non-tumorigenic prostatic epithelial RWPE-1 cells were used in a 3-D in vitro cell model. Upon immunostaining, acini formation was detected in different RWPE cell models, and the cell polarity and central lumen were distinctly different from that seen under 2-D culture conditions. Treatment with IR caused the disruption of acinar structures in a dose-dependent manner. The lower dose of 2 Gy retarded formation of the spheroid structure, but cell clusters were still obvious; however, a dose of 5 Gy caused the cells to detach from each other and undergo cell death, suggesting that IR might intervene somehow in the formation of acini. However, the underlying mechanism remains to be revealed.

To determine whether autophagy, or type II programmed cell death, participates in the process of IR-induced disruption of acini, we chose autophagy-associated genes such as Beclin 1, ATG7, and Akt. Beclin and ATG7 were reported to positively regulate autophagy $[9,11,12]$, whereas Akt suppresses development of autophagy $[18,19]$. Our preliminary studies 


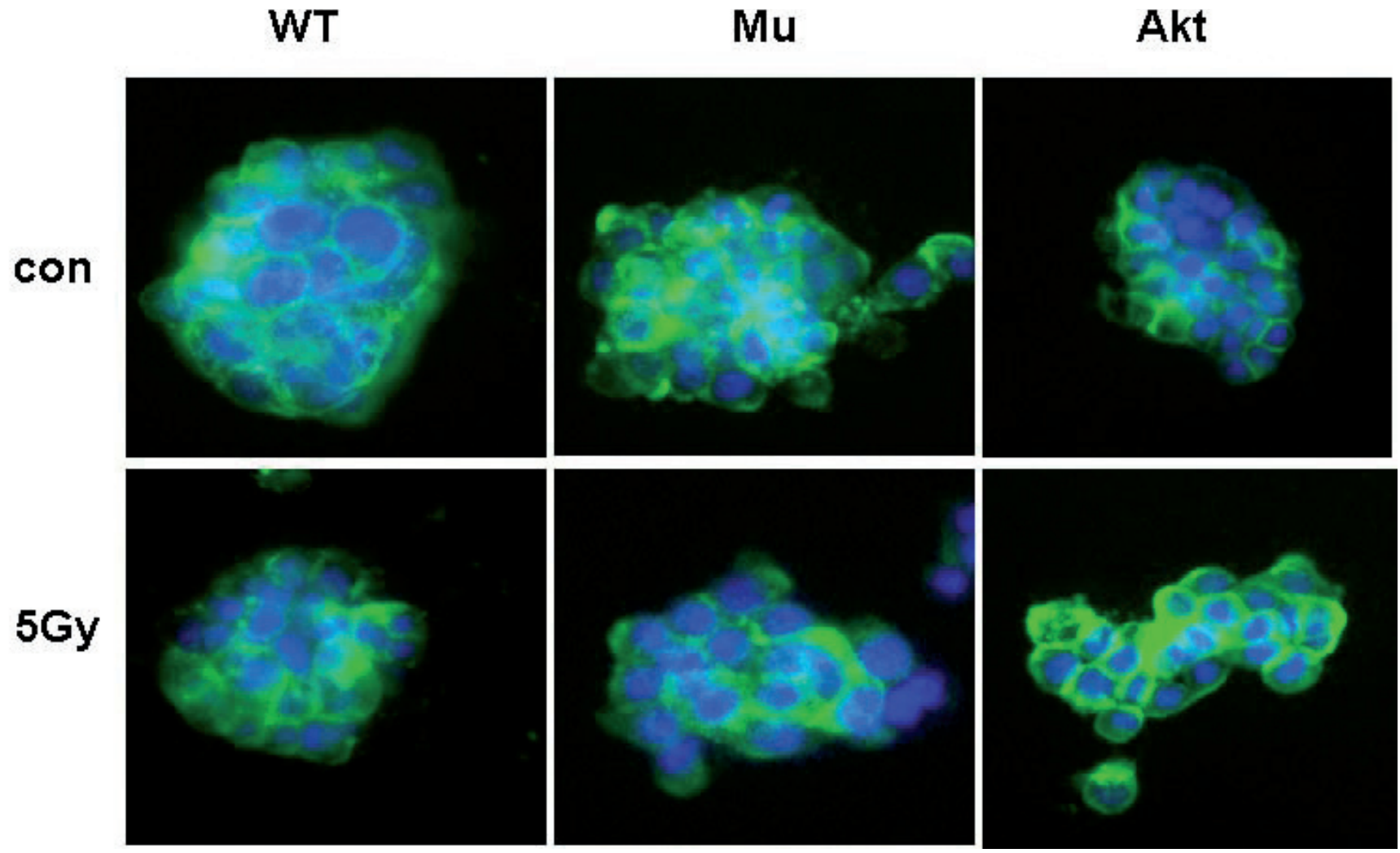

Figure 10. Morphological changes in Akt overexpression RWPE cells by radiation under 3-D culture conditions. Cells were fixed on the $10^{\text {th }}$ day of growth and stained and examined by immunofluorescence microscopy for nuclei detection (blue) and integrin (green) expression. Compared with the formation of acini in wild-type RWPE cells, the transfected cells exhibited a disorganized structure. Although the cells clustered together, normal acini were not observed in Akt-transfected or Mu-transfected cells. After $5 \mathrm{~Gy}$ of radiation, cells detached from each other, but still had smooth edges, and no cell death occurred in both Akt-transfected and Mu-transfected cells.

indicated that IR might induce expression of Beclin and facilitate autophagic cell death in MCF-10A cells (data not shown). Thus, we tried to prove the functions of Beclin 1 and ATG7 conversely: Beclin 1 and ATG7 were knocked down, and Akt was overexpressed in RWPE- 1 cells, respectively.

Roles of Beclin 1 and ATG7 in the process of autophagy and acinar morphogenesis. MAP-LC3 is a specific marker for autophagy and the Beclin gene is used as well to indirectly detect autophagic status. After radiation, aggregation of MAP-LC3 in the autophagosome was visible in the cytosol, and a time-dependent increase in Beclin and MAP-LC3 expression was shown. Beclin-shRNA and ATG7-shRNA were then introduced into RWPE-1 cells to establish Beclin ${ }^{-1-}$ and ATG7 ${ }^{-1}$ cell models. Morphological changes in Beclin-RNAi cells were distinct: cells became rounded and the extension of twigs disappeared, compared with wild-type RWPE-1 cells. However, ATG7-RNAi cells maintained the normal elongating morphological structure. Both Beclin-RNAi and ATG7-RNAi led to increase in cell rapid growth and proliferation, indicating growth suppression of autophagy in RWPE- 1 cells. Radiation decreased the colony numbers in Beclin-RNAi cells (15\% of sham-radiation for Beclin-RNAi cells vs. $25 \%$ of sham- radiation for control cells) and the surviving fraction curve showed that the slope in the Beclin-RNAi group was greater than that of the wild-type control, suggesting radiosensitization of Beclin-RNAi. Radiation led to G1 arrest, G2 arrest, and $\mathrm{S}$ phase decrease, consistent with the radiobiological rules that radiation induces G1 and G2 arrest and provides an opportunity to repair damaged DNA. However, in the Beclin-RNAi group, radiation triggered G1 decrease, G2 arrest, and $\mathrm{S}$ phase extension, suggesting the lack of autophagy resulting from Beclin-RNAi that might cause deletion of the G1 checkpoint. Consequently, cells went into $S$ phase and the replication of abnormal DNA in S phase and accomplishment of cycle progression through G2/M phase might lead to genome instability.

Radiation altered also the expression of different proteins, and an increase in Beclin1 and p21 by IR in wild-type cells was detected. After introduction of Beclin-shRNA or ATG7-shRNA by retroviral vectors, suppression of Beclin 1 and an increase in p21 occurred, whereas no change in p53 was observed, consistent with the immortalization of RWPE cells by HPV E6/E7 inevitably resulting in the degradation of p53 and pRb. Beclin 1 showed a more significant increase in 
Beclin-RNAi cells than in wild-type cells after both 24- and 48-h IR treatment, and only increased in ATG-RNAi cells at $24 \mathrm{~h}$, whereas p21 displayed the same change as in wild-type cells. Together with our previous data that revealed marked induction of Beclin1 in MCF-10A cells by radiation (data not shown), these data suggest that radiation might affect the process of autophagy, at least by facilitating expression of Beclin 1. In the present study, 5-FU was also used as a chemical control to compare with the physical stimulus of radiation.

Morphological changes in gene knock-down RWPE cells by radiation under 3-D culture conditions were detected by immunostaining and fluorescence microscopy. Wild-type RWPE cells aggregated and formed acini under 3-D culture conditions with obvious cell polarity, whereas in Beclin-RNAi and ATG7-RNAi cells, although the cells also clustered together, normal acini were not observed. After treatment with radiation, cells detached from each other, and cell death was more obvious in Beclin-RNAi and ATG7-RNAi cells than in wild-type cells, consistent with the increase in radiosensitivity in Beclin-RNAi cells under 2-D culture conditions. This finding suggests that disruption of the autophagy process blocks acinar morphogenesis under 3-D culture conditions.

Roles of Akt in the process of autophagy and acinar morphogenesis. The Akt gene, reported to be a negative regulator of autophagy that functions in a manner in contrast to that of Beclin and ATG7 [18,19], has been shown to play critical roles in regulation of multiple cellular functions such as transcription, cell survival, cell cycle progression, angiogenesis, and cell motility - all of which are important to the malignant process. In this study, the Akt gene (Wt-Akt) and mutant Akt (179M, $\mathrm{Mu}$-Akt) were introduced into RWPE cells, respectively. The morphological characteristics, protein expression, and cell cycle progression under both 2-D and 3-D culture conditions were observed.

RWPE cells with the introduction of Wt-Akt elongated greatly and formed microspike-like structures. The presence of $\mathrm{Mu}$-Akt altered the morphology and microspikes were still formed, albeit to a lesser extent. The growth curve and colony formation assay demonstrated that cells transfected with Akt or Akt mutant genes grew faster than wild-type and empty vector-infected cells, illustrating that $A k t$ functions as a growth-related gene in RWPE cells. Cell cycle progression revealed that a lower dose of radiation (2 Gy) caused G1 arrest, while a higher dose (5 Gy) caused G2 arrest, and no difference was found between the control and Akt overexpression groups, demonstrating no role of Akt in the cell cycle here. The protein expression profile showed that constitutive expression of Akt was weak in the control group, while the introduction of either Wt-Akt or Mu-Akt increased the expression of Akt dramatically. Phospho-Akt was detected only in cells containing Wt-Akt and was unaltered in the presence or absence of radiation, suggesting that IR has little effect on Akt under 2-D culture conditions.

The morphological structure under 3-D culture conditions illustrated the formation of typical acini in wild-type RWPE cells, whereas cells in the Wt-Akt or Mu-Akt group were able to cluster together but did not form acini. After $5 \mathrm{~Gy}$ of radiation, cells detached from each other, but no cell death was detected in either Wt-Akt- or Mu-Akt-infected cells. Cells seemed to be more resistant to radiation after transfection of Akt. IR has been previously reported to facilitate changes in $\mathrm{p} 53$ that might indirectly suppres mTOR and Akt, consequently contributing to the process of autophagy $[13,18-20]$. Our data here showed inconsistency with these previous results: the IR-induced phosphorylation of Akt might result from degradation of p53 by HPV E6/E7 in RWPE cells, since the inactivation of $\mathrm{p} 53$ would release the suppression on Akt. Given that the autophagic event was too complicated to be explained by a single factor, possible mechanisms should be further discussed.

In summary, our results show the effects of radiation on acini formation under 3-D culture conditions. This ductal formation is disrupted by ionizing radiation due to gene and protein profile alterations. Importantly, compromised 3-D growth control of mammary epithelial cells under different genetic backgrounds might render them responsive to radiation. Thus, our results reveal an important interplay between genetic and epigenetic (tumor environment) alterations in prostatic epithelial cells. Our data also suggest the possible role of autophagy-related genes in IR-induced disruption of 3-D spheroid structures; all these effects can be attributed to deregulated cell-cell and cell-matrix interactions caused by radiation. Future studies to determine whether any other factors participate in this regulation and how they function are warranted.

Acknowledgements: We acknowledge the expert technical support of Ms. Elke Pravda for confocal microscopic imaging at Children's Hospital of Boston. This study was completed partly at the Harvard School of Public Health and partly at Jilin University, and was supported by NSFC grant from China (30770649, 30970682).

\section{References}

[1] KOGAN I, GOLDFINGER N, MILYAVSKY M, COHEN M, SHATS I. et al. hTERT-immortalized prostate epithelial and stromal-derived cells: an authentic in vitro model for differentiation and carcinogenesis. Cancer Res. 2006; 66: 3531-3540. http://dx.doi.org/10.1158/0008-5472.CAN-05-2183

[2] BELLO-DEOCAMPO D, KLEINMAN HK, WEBBER MM. The role of alpha 6 beta 1 integrin and EGF in normal and malignant acinar morphogenesis of human prostatic epithelial cells. Mutat Res. 2001;480-481:209-217. http://dx.doi. org/10.1016/S0027-5107(01)00201-9

[3] BISSELL MJ, RADISKY D. Putting tumours in context. Nat Rev Cancer. 2001; 1: 46-54. http://dx.doi.org/10.1038/35094059

[4] JACKS T, WEINBERG RA. Taking the study of cancer cell survival to a new dimension Cell. 2002; 111: 923-925 http:// dx.doi.org/10.1016/S0092-8674(02)01229-1

[5] SCHMEICHEL KL, BISSELL MJ. Modeling tissue-specific signaling and organ function in three dimensions. J Cell Sci. 2003; 116: 2377-2388. http://dx.doi.org/10.1242/jcs.00503 
[6] ABBOTT A. Cell culture: biology's new dimension. Nature. 2003; 424: 870-872. http://dx.doi.org/10.1038/424870a

[7] BELLO D, WEBBER MM, KLEINMAN HK, WARTINGER DD, RHIM JS. Androgen responsive adult human prostatic epithelial cell lines immortalized by human papillomavirus 18.Carcinogenesis. 1997; 18: 1215-1223. http://dx.doi. org/10.1093/carcin/18.6.1215

[8] DEBNATH J, MUTHUSWAMY SK, BRUGGE JS. Morphogenesis and oncogenesis of MCF-10A mammary epithelial acini grown in three-dimensional basement membrane cultures. Methods. 2003; 30: 256-268. http://dx.doi.org/10.1016/S10462023(03)00032-X

[9] YU L, ALVA A, SU H, DUTT P, FREUNDT E. et al.Regulation of an ATG7-beclin 1 program of autophagic cell death by caspase-8. Science. 2004; 304: 1500-1502. http://dx.doi. org/10.1126/science. 1096645

[10] LANG SH, STARK M, COLLINS A, PAUL AB, STOWER MJ. et al. Experimental prostate epithelial morphogenesis in response to stroma and three-dimensional matrigel culture. Cell Growth Differ. 2001; 12: 631-640.

[11] YUE ZY, JIN SK, YANG CW, LEVINE A J, HEINTZ N. Beclin 1, an autophagy gene essential for early embryonic development, is a haploinsufficient tumor suppressor. PNAS, 2003, 100: 15077-15082. http://dx.doi.org/10.1073/ pnas. 2436255100

[12] YANG YP, LIANG ZQ, GU ZL, QIN ZH. Molecular mechanism and regulation of autophagy. Acta Pharmacologica Sinica 2005; 26: 1421-1434. http://dx.doi.org/10.1111/j.17457254.2005.00235.x

[13] ELLINGTON AA, BERHOW MA, SINGLETARY KW. Inhibition of Akt signaling and enhanced ERK1/2 activity are involved in induction of macroautophagy by triterpenoid B-group soyasaponins in colon cancer cells.Carcinogenesis. 2006; 27: 298-306. http://dx.doi.org/10.1093/carcin/bgi214
[14] KANZAWA T, IWADO E, AOKI H, IWAMARU A, HOLLINGSWORTH EF. et al. Ionizing radiation induces apoptosis and inhibits neuronal differentiation in rat neural stem cells via the c-Jun NH2-terminal kinase (JNK) pathway. Oncogene. 2006; 25: 3638-48. http://dx.doi.org/10.1038/ sj.onc. 1209414

[15] GONG SL, LIU SC, LIU JX, ZHANG YC, LIU SZ. Adaptive response of thymocyte apoptosis and cell cycle progression induced by low dose X-ray irradiation in mice. Biomed Environ Sci. 2000; 13: 180-188.

[16] ROSSI L, REVERBERI D, PODESTA G, LASTRAIOLI S, CORVO R. Co-culture with human fibroblasts increases the radiosensitivity of MCF-7 mammary cancinoma cells in collagen gels. Int. J. Cancer. 2000; 85, 667-673. http://dx.doi. org/10.1002/(SICI) 1097-0215(20000301)85:5<667::AIDIJC12>3.0.CO;2-1

[17] TANAKA R, SAITO T, ASHIHARA K, NISHIMURA M, MIZUMOTO $\mathrm{H}$. et al. Three-dimensional coculture of endometrial cancer cells and fibroblasts in human placenta derived collagen sponges and expression matrix metalloproteinases in these cells. Gynecologic Oncology. 2003; 90, 297-304. http://dx.doi.org/10.1016/S0090-8258(03)00335-4

[18] LEVINE AJ, FENG ZH, MAK TW, YOU H, JIN SK. Coordination and communication between the p53 and IGF1-AKT-TOR signal transduction pathways. Genes \& Dev. 2006; 20, 267-275. http://dx.doi.org/10.1101/gad.1363206

[19] SHINOHARA ET, CAO C, NIERMANN K, MU Y, ZENG $\mathrm{FH}$. et al. Enhanced radiation damage of tumor vasculature by mTOR inhibitors. Oncogene. 2005; 24, 5414-5422. http:// dx.doi.org/10.1038/sj.onc.1208715

[20] FENG ZH, ZHANG HY, LEVINE AJ, JIN SK. The coordinate regulation of the p53 and mTOR pathways in cells. PNAS. 2005; 102, 8204-8209. http://dx.doi.org/10.1073/ pnas. 0502857102 